\title{
TIEMPO DE COMPROMISO MOTOR EN EDUCACIÓN PRIMARIA
}

\author{
MOTOR COMMITMENT TIME IN PRIMARY EDUCATION
}

\author{
Natalia Fernández González ${ }^{1}$ \\ ${ }^{1}$ Graduada Universidad de Málaga, Málaga (España)
}

\author{
Detalles del artículo: \\ Número de palabras: 3500; Tablas: 3; Figuras: 1; Referencias: 25 \\ Recibido: febrero 2019; mayo 2019; Publicado: julio 2019 \\ Conflicto de interés: El autor declara que no existen conflictos de interés. \\ Correspondencia del autor: Natalia Fernández González, nataliafergon1997@gmail.com
}

\section{Resumen}

Introducción: el tiempo de compromiso motor según Gómez y Sánchez (2014), es aquel tiempo en el que el alumnado lo dedica a una actividad motora, considerado como medidor de la eficacia docente en cuanto es el tiempo en el que más aprendizaje se producen en una clase de Educación Física. Material y método: en este trabajo el objetivo principal ha sido valorar el tiempo de compromiso motor de los discentes, de modo que he registrado una muestra de 20 alumnos del primer ciclo de educación primaria a lo largo de 4 semanas ( 1 mes), a distintas horas del día. La herramienta utilizada ha sido un cronómetro y una hoja de registro donde se recogían los momentos de actividad motriz de los alumnos durante la sesión. Resultados: se ha obtenido una media del 54,29\% del tiempo de compromiso motor en las sesiones de educación física. Conclusiones: el tiempo de compromiso motor de nuestro estudio es bajo, siendo mayor en la sesión tras el recreo y menor durante la primera sesión de la mañana.

Palabras claves: tiempo, Educación Física, actividad física, sesiones, discentes.

\section{Abstract}

Introduction: the motor commitment time according to Gómez and Sánchez (2014), is that time in which the students dedicate it to a motor activity, considered as a measure of teacher effectiveness as it is the time in which more learning occurs in a class of Physical Education. Material and methods: in this work the main objective has been to assess the time of motor commitment of the students, so I have registered a sample of 20 students of the first cycle of primary education over 4 weeks ( 1 month), at different times of the day. The tool used was a chronometer and a record sheet where the moments of motor activity of the students during the session were collected. Results: we obtained an average of $54.29 \%$ of motor engagement time in physical education sessions. Conclusions: it has been concluded that the motor commitment time is much higher in the session after recess, with the shortest time during the first session.

Key words: time, Physical Education, physical activity, sessions, students. 


\section{INTRODUCCIÓN}

En la actualidad, es cada vez más habitual que haya problemas de salud dirigidos tanto a niños como a jóvenes. Uno de los factores influyentes son la aparición de las nuevas tecnologías, así como el cambio de hábito en la vida de cualquier persona de nuestra sociedad actual. Esto está desembocando en un incremento de la inactividad física y sedentarismo, apareciendo enfermedades cardiovasculares, diabetes, obesidad, etc., (Fernández-Revelles, 2008).

La literatura científica encuentra una fuerte relación entre sedentarismo y obesidad, incluso entre ejercicio y salud. De acuerdo con la OMS (2000), la obesidad es una enfermedad crónica, que se caracteriza por el incremento de la grasa corporal y que está asociada a mayor riesgo para nuestra salud. En un país como el nuestro, un país desarrollado, es una enfermedad muy habitual, por lo que se considera muy grave para estar en el siglo en el que nos encontramos. En relación con este tema, está ligado la obesidad infantil, afectando mucho a las escuelas en la actualidad. Según un estudio sobre la obesidad tanto juvenil como infantil llevado a cabo por Sánchez-Cruz, Jiménez-Moleón, Fernández-Quesada y Sánchez (2013), nos muestra unos porcentajes bastante altos de niños y niñas con obesidad y sobrepeso, siendo esta un $40 \%$. Es un dato muy alarmante y que nosotros como futuros docentes en la mención de educación física tenemos una gran responsabilidad, por ello el tiempo de compromiso motor en una clase es muy importante, ya que realmente es el tiempo en el que el discente está en continuo movimiento. Además, muchos niños y niñas en estas edades, el único ejercicio físico que hace es el de la clase de educación física, por ello tenemos esta gran responsabilidad de concienciar a nuestros discentes de la gran importancia y el papel tan importante que desempeña el ejercicio físico. Un estudio realizado por Ceballos-Tellez, Romero-Ramos y Prieto (2018), afirma que aquellos estudiantes con un peso saludable tienen una buena condición física frente a aquellos que tienen sobrepeso u obesidad, afectando a su salud.

Pues bien, las personas comprendidas entre las edades de 5-17 años, según la OMS (2010), tienen que comprometerse a realizar cualquier actividad física una hora diaria, ya sea de intensidad baja o media. De esta manera podremos reducir el gran porcentaje de personas con sobrepeso u obesidad que hay sobre todo en edades tempranas. Según nos indica Revelles (2008), estos problemas de sedentarismo, malos hábitos alimenticios y la inactividad física, incidían principalmente en la población adulta, pero desde hace una década el problema se está dando más en los niños y adolescentes.

Tras varios estudios realizados por (Olds, Tomkinson, Leger \& Cazorla, 2006; Ruiz et al., 2009), nos advierte que existe relación entre la actividad/actitud física no realizada en la infancia con futuras enfermedades de salud, tal y como enfermedades mentales, respiratorias, $\mathrm{y}$ todas aquellas enfermedades mencionadas con anterioridad.

Según el Real Decreto 126/2014, de 28 de febrero, por el que se establece el currículo básico de la Educación Primaria (BOE, 2014), asegura que un 80\% de estudiantes en edad escolar, practican deporte únicamente en las horas de Educación Física, siendo el 20\% restante, aquellos alumnos y alumnas que practican algún deporte fuera del ámbito escolar. Por estas cifras, es importante el rol que juega el o la docente en una sesión de Educación Física. Del mismo modo, se realizan únicamente dos sesiones semanales de 45 minutos cada una, en cualquier curso de la etapa de educación primaria, siendo una cifra bastante baja en comparación con las demás asignaturas que se imparten en la escuela. Además, tras varios estudios realizados por (Koplan, Liverman \& Kraak, 2005; Pate et al., 2006), nos argumentan 
que debemos impartir un mínimo de 150 minutos semanales de educación física, es decir, más del triple de minutos que se da actualmente en cualquier centro.

Después de que varias personas realizaran varios estudios, se puede comprobar que el tiempo de compromiso motor de los discentes en las clases de Educación Física, no tiene mucha relación con el tiempo de la práctica teórica (Olmedo, 1998). Por lo que el tiempo de compromiso motor tiene relación con las particularidades del medio físico, el mal o buen estado que tienen las instalaciones del centro, la disponibilidad (Sallis et al., 2001), la estructura y organización de las actividades, la motivación previa de los discentes (Solmon, 2003; Calderón \& Palao, 2005; Silverman, 2005), y el modelo de sesión que se ha planteado con anterioridad (Olmedo, 1998). Por todo esto, según Yanci Irigoyen, J., Vinuesa Mendieta, A., y Yanci Irigoyen, L. (2016), nos indica que es importante analizar el tiempo de compromiso motor (TCM) de los discentes en las sesiones de Educación Física en relación con la organización, ya sea la hora en la que se imparte la sesión, el modelo de actividad, etc. Por lo tanto, en este trabajo de investigación, se analizará si el TCM tiene relación y varía con respecto a las diferentes horas del día, y si también tiene que ver con el tipo de actividad que se está desempeñando, el tipo de agrupamiento de los discentes para realizar las actividades propuestas, etc.

Aunque por norma general una sesión de Educación Física tenga una duración de 45 minutos, según nos comenta Sánchez (1986), de una sesión de 1 hora, únicamente 20-25 minutos son los que se rinden realmente. Esta cifra tiene relación con los datos obtenidos en mi hoja de registro.

El tiempo de compromiso motor de los discentes seleccionados del centro en el que he estado, se ha basado en los siguientes ya recogidos en Fernández (2019):

Estilos de enseñanza (E. E.):_Como definición de este término, Delgado (1991) nos asegura que es la forma particular de interaccionar con el alumnado, poniéndose de manifiesto en las decisiones preactivas, interactivas y postactivas de aquellas interacciones que se dan. No existe un estilo de enseñanza perfecto ni puro, ya que son adaptados a cada tipo de docente, alumnado, objetivos propuestos, etc. Lo más importante es que el docente sea capaz de utilizar el E.E. apropiado en el momento indicado. Refiriéndonos al caso más particular que estamos investigando, el estilo de enseñanza utilizado mientras hemos estado analizando el tiempo de compromiso motor ha sido el estilo tradicional Delgado (1991), modificando el mando directo. El papel del docente ha sido el de autoridad, en el que previamente ha planificado la clase (decisión preactiva), posteriormente en clase ha llevado a cabo su planificación (decisión interactiva) y finalmente los discentes han sido evaluados por el docente (decisión postactiva). En las sesiones se ha dado una comunicación unidireccional, mientras que los discentes tenían el rol de dar respuestas y atender.

Técnicas de enseñanza (T. E.): Noguera (1993), nos afirma que las técnicas de enseñanza se refieren a la interacción de carácter comunicativo de todo aquello que se pretende difundir y técnico. Tienen como objetivo principal el comportamiento del docente en relación con la manera de transmitir la información durante la sesión, también la comunicación didáctica y por último exponer las actividades que deben llevar a cabo los docentes. Basándonos en esta investigación, la técnica de enseñanza más utilizada ha sido la instrucción directa, teniendo como objetivo principal el tener que transmitir a los dicentes la actividad física que deben realizar por parte del docente. Según nos asegura Contreras (2010), para que la información se transmita con mayor eficacia, tenemos que utilizar la información tanto visual como verbal. La muestra al ser alumnos del primer ciclo, la información visual es fundamental, ya que así los 
alumnos observan a través de una demostración del docente o de un compañero de clase, la actividad que deben realizar. Por otra parte, la información verbal también es muy importante ya que se le debe transmitir la información de forma concisa y muy clara.

- Estrategias en la práctica (E.P.): Noguera (1993) nos define estrategia en la práctica como la manera en la que las actividades se van a realizar, en las que se componen por una progresión de la enseñanza de una habilidad motriz concreta.

Tras un estudio realizado por Campos, Garrido y Castañeda (2011), en el que investigaron el estilo de enseñanza como determinante del tiempo de compromiso motor, expone que, mediante el estilo de enseñanza descubrimiento guiado, el tiempo de compromiso motor es mayor. Por lo tanto, pone de manifiesto que este estilo de enseñanza es mucho más eficaz y se pueden lograr de manera más cómoda los objetivos propuesto en la sesión.

\section{MATERIAL Y MÉTODOS}

\section{Participantes}

La muestra que se ha seleccionado para este trabajo de investigación ha sido escogida al azar en un colegio público situado en la provincia de Málaga denominado C.E.I.P. Ramón Simonet. Los discentes escogidos para la muestra han sido del primer ciclo de educación primaria, es decir, primero y segundo de primaria. Son discentes tanto de $1^{\circ}$ como de $2^{\circ}$ de educación primaria. Se ha escogido a los participantes de forma aleatoria.

\section{Materiales}

En relación con los instrumentos se ha utilizado el cronómetro para contabilizar el tiempo en el que el discente estaba en movimiento y una hoja de registro (Tabla 1).

Tabla 1. Hoja de registro de los datos obtenidos

\begin{tabular}{|l|l|l|l|l|l|l|l|l|l|}
\hline Fecha & & Hora & & Contenido & & Grupo & & TCM & \\
\hline Fecha & & Hora & & Contenido & & Grupo & & TCM & \\
\hline Fecha & & Hora & & Contenido & & Grupo & & TCM & \\
\hline Fecha & & Hora & & Contenido & & Grupo & & TCM & \\
\hline
\end{tabular}

\section{Procedimiento}

Los datos se informan como media (desviación estándar). Se ha realizado un análisis descriptivo para examinar las diferencias de medias entre las diferentes sesiones a lo largo del día. Todos los análisis estadísticos se realizaron utilizando la versión 25.0 de SPSS para Windows (IBM ${ }^{\circledR}$ SPSS ${ }^{\circledR}$ Statistics). Los datos obtenidos han sido recogidos en un centro educativo de Primaria de la provincia de Málaga. Los contenidos de las sesiones impartidas han sido de desplazamientos, salto y giro, aplicando las mismas actividades, o con alguna pequeña variación dependiendo del ciclo. 


\section{RESULTADOS}

Los resultados obtenidos del tiempo de compromiso motor en los discentes del primer ciclo de educación primaria se pueden observar en la tabla 2.

Tabla 2. Resultados obtenidos del Tiempo de Compromiso Motor

\begin{tabular}{|c|c|c|c|c|c|}
\hline Fecha & Hora & Unidad Didáctica & Grupo & $\begin{array}{l}\text { Minutos de } \\
\text { TCM }\end{array}$ & Sexo \\
\hline Lunes 21 & $09: 45-10: 30$ & Desplazamientos & $1^{\circ} \mathrm{A}$ & 19,5 & Niño \\
\hline Martes 22 & $10: 30-11: 15$ & Desplazamientos & $1^{\circ} \mathrm{D}$ & 19 & Niño \\
\hline Miércoles 23 & 09:00-09:45 & Desplazamientos & $1^{\circ} \mathrm{C}$ & 17 & Niña \\
\hline Jueves 24 & $13: 15-14: 00$ & Desplazamientos & $2^{\circ} \mathrm{D}$ & 20 & Niña \\
\hline Viernes 25 & $11: 45-12: 30$ & Desplazamientos & $1^{\circ} \mathrm{D}$ & 21,5 & Niño \\
\hline Lunes 28 & $11: 45-12: 30$ & Salto y giro & $1^{\circ} \mathrm{A}$ & 20 & Niño \\
\hline Martes 29 & $10: 30-11: 15$ & Salto y giro & $1^{\circ} \mathrm{D}$ & 19,5 & Niña \\
\hline Miércoles 30 & 09:00-09:45 & Salto y giro & $1^{\circ} \mathrm{C}$ & 18 & Niña \\
\hline Jueves 31 & $13: 15-14: 00$ & Salto y giro & $2^{\circ} \mathrm{D}$ & 21 & Niño \\
\hline Viernes 1 & $11: 45-12: 30$ & Salto y giro & $1^{\circ} \mathrm{D}$ & 22,5 & Niño \\
\hline Lunes 4 & $11: 45-12: 30$ & Salto y giro & $1^{\circ} \mathrm{A}$ & 19 & Niña \\
\hline Martes 5 & $10: 30-11: 15$ & Salto y giro & $1^{\circ} \mathrm{D}$ & 19,5 & Niña \\
\hline Miércoles 6 & 09:00-09:45 & Salto y giro & $1^{\circ} \mathrm{C}$ & 17,5 & Niño \\
\hline Jueves 7 & $13: 15-14: 00$ & Salto y giro & $2^{\circ} \mathrm{D}$ & 19,5 & Niño \\
\hline Viernes 8 & $11: 45-12: 30$ & Salto y giro & $1^{\circ} \mathrm{D}$ & 22 & Niña \\
\hline Lunes 11 & $11: 45-12: 30$ & Salto y giro & $1^{\circ} \mathrm{A}$ & 19,5 & Niña \\
\hline Martes 12 & $10: 30-11: 15$ & Salto y giro & $1^{\circ} \mathrm{D}$ & 19,5 & Niño \\
\hline Miércoles 13 & 09:00-09:45 & Salto y giro & $1^{\circ} \mathrm{C}$ & 17 & Niño \\
\hline Jueves 14 & $13: 15-14: 00$ & Salto y giro & $2^{\circ} \mathrm{D}$ & 18,5 & Niña \\
\hline Viernes 15 & $11: 45-12: 30$ & Salto y giro & $1^{\circ} \mathrm{D}$ & 21 & Niña \\
\hline
\end{tabular}

(*) Datos recogidos a lo largo del mes de enero-febrero, tiempo de compromiso motor expresado en minutos

La media del tiempo de compromiso motor ha sido de un $43,44 \%$ del tiempo total de clase, es decir, el tiempo que verdaderamente están los discentes en movimiento sería una media de 19,55 minutos, de los 45 minutos que dura una sesión de Educación Física. 
Se podría observar que en las sesiones impartidas a primera hora de la mañana, hay un tiempo de compromiso mucho menor que en el resto de sesiones, puesto que se pierde más tiempo al subir a clase, soltar las mochilas y bajar al patio. Por el contrario, en aquellas sesiones que se realizan después del recreo, el tiempo de compromiso motor es mayor.

En la figura 1 se puede observar la media de tiempo de compromiso motor expresada según las distintas horas del día en que se lleva a cabo la sesión de Educación Física:

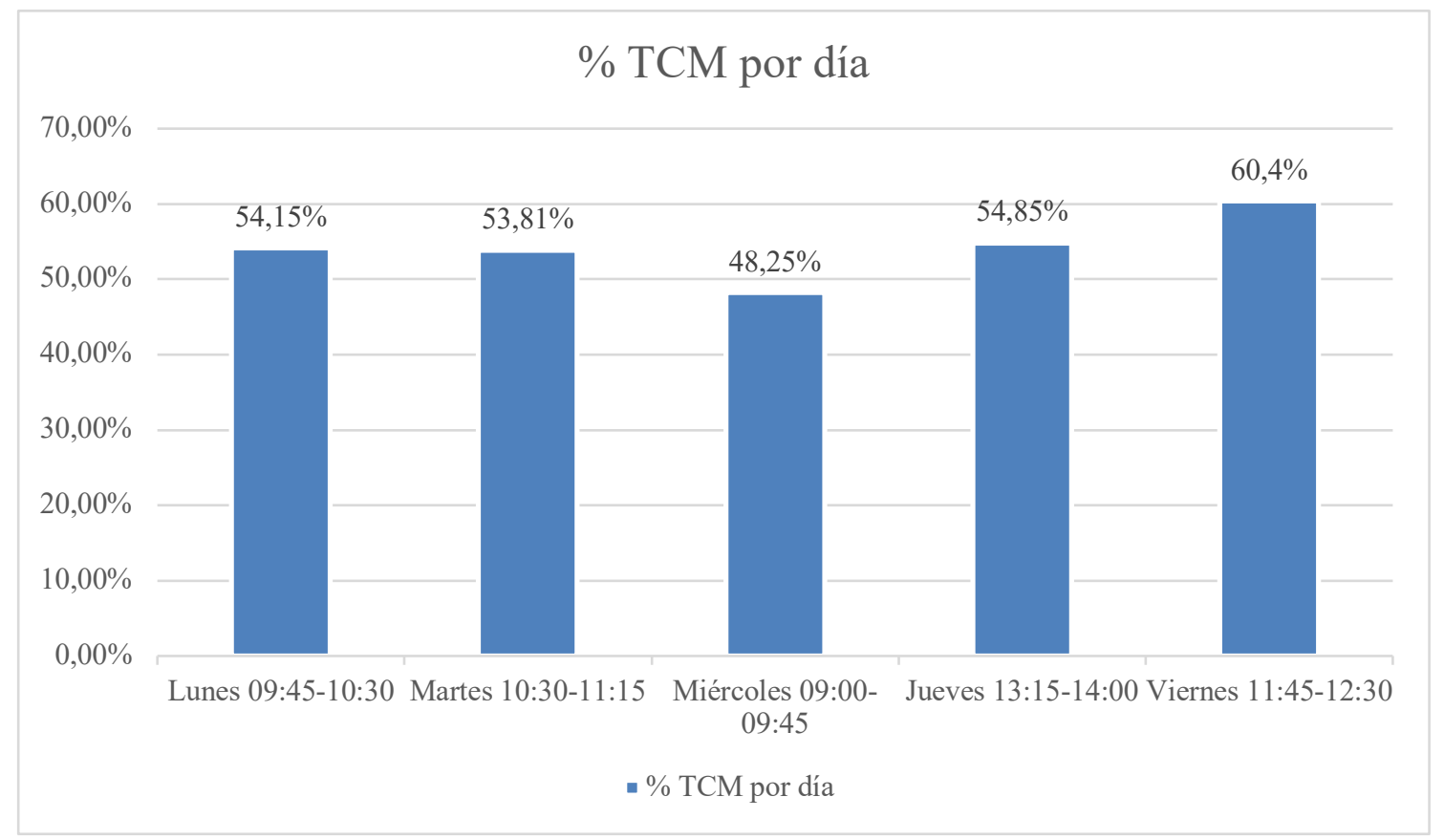

Figura 1. Porcentaje del TCM según las distintas horas del día en que se lleva a cabo la sesión de Educación Física

Se puede observar en la figura anterior que el porcentaje del TCM es mayor en el horario de las 11:45 hasta las 12:30, es decir, después del recreo. Con respecto a las demás franjas horarias todas mantienen unos resultados similares, exceptuando el horario de 09:00-09:45, es decir, en la primera sesión de Educación Física de la mañana.

Tabla 3. Resultados obtenidos del Tiempo de Compromiso Motor según el tipo de habilidad

\begin{tabular}{lr|c} 
Tipo habilidad & Tiempo (min.) & SD \\
\hline Desplazamiento & 19,400 & 1,6355 \\
\hline Salto/Giro & 19,600 & 1,5492 \\
\hline
\end{tabular}

Según los datos de la tabla 3, se han realizado dos tipos de habilidades, el desplazamiento y el salto y giro, obteniéndose unos datos similares en las medias de ambas habilidades. 


\section{CONCLUSIONES}

Se ha registrado un tiempo de compromiso motor mayor en el horario de 11:45 a 12:30, tras el recreo, ya que no se pierde tiempo en ir a la clase a por ellos, que se coloquen en fila y bajar. En la sesión que se pierde más tiempo y por lo tanto el TCM es menor, es de 09:00 a 09:45, en la primera sesión de Educación Física, influyendo aquí la entrada al colegio. Las demás sesiones tienen unos resultados similares, al igual que los resultados por género, no existiendo diferencia de TCM en ninguno de los casos.

A modo de conclusión, Piéron (2005) nos dice que una de las competencias que más influyen en el proceso de enseñanza-aprendizaje es la correcta gestión del tiempo de clase. El TCM es uno de los factores que más ligado está a esta gestión, garantizando un buen aprendizaje por parte de los discentes. Por ello, hay que valorar el TCM en educación primaria, hay que conseguir que sea lo mayor posible. En este trabajo de investigación se ha podido observar como no se ha llegado ni a un 70\% de TCM en clases del primer ciclo de educación primaria. Esto es un dato que nos tendría que hacer reflexionar sobre el tiempo que deberían estar físicamente activos nuestros escolares, entendiendo que el TCM de nuestras sesiones de Educación Física debería ser mayor.

\section{REFERENCIAS BIBLIOGRÁFICAS}

BOE. (2014). Real Decreto 126/2014, de 28 de febrero, por el que se establece el currículo básico de la Educación Primaria. Consultado el 3 de abril de 2019. http://www.boe.es/boe/dias/2014/03/01/pdfs/BOE-A-20142222.pdf

Calderón, A., \& Palao, J.M. (2005). Incidencia de la forma de organización en la sesión sobre el tiempo de práctica y le percepción de la motivación en el aprendizaje de las habilidades atléticas. Apunts Educación Física y Deportes, 81, 29-37.

Ceballos-Tellez, D., Romero-Ramos, O., \& Prieto, F. J. R. (2018). Relationship of BMI and physical condition in schools from 6 to 8 years of age. La revista rusa de educación fisica y el deporte (Vol. 13).

Contreras Jordán, O. R., Gil Madrona, P., Sebastiani Obrador, E., Pascual Baños, C., Huguet Mora, D., Hernández Álvarez, J. L., ... \& Capllonch Bujosa, M. (2010). Didáctica de la educación física (Vol. 2). Ministerio de Educación.

Delgado, M. A. (1991). Los estilos de enseñanza en la Educación Física. Propuesta para una Reforma de la Enseñanza. Granada: ICE de la Universidad de Granada.

Fernández González, N. (2019). Portafolio del Prácticum III.II del Grado en Educación Primaria. Manuscrito no publicado, Facultad de Ciencias de la Educación, Universidad de Málaga, España.

Fernández-Revelles, A. B. (2008). El tiempo en la clase de Educación Física: la competencia docente tiempo. Deporte y actividad física para todos, (4), 102-120. Ferreira, F.S., Mota, J. y Duarte, J.A. (2014). Patterns of physical activity in Portuguese adolescents. Evaluation during physical education clases through accelerometry. Archives of Exercise in Health and Disease, 4 (2), 280-285. doi: 10.5628/aehd.v4i2.135.

Gómez-Mármol, A., \& Sánchez-Alcaraz, B. J. (2014). Influencia de la técnica de enseñanza sobre el tiempo de compromiso motor en las clases de Educación Física, el esfuerzo y la diversión percibida. In VII Congreso Internacional de la Asociación Española de Ciencias del Deporte. Cáceres.

Koplan, J., Liverman, C.T., \& Kraak, V.I. (2005). Preventing Childhood Obesity: Health in the Balance. National Academy Press, Washington, DC.

Muradas, M. \& Porta, M (2007). Las memorias del Practicum I de la titulación de Maestros de Educación Infantil: sobre qué reflexionan los alumnos, en A. Cid y otros (coords.) Buenas prácticas en el Practicum. Santiago de Compostela. Imprenta Universitaria. 977-991. 
Noguera, D. (1993). MA Pedagogía aplicada al deporte. Texto del Curso de Acceso al Máster en Alto Rendimiento Deportivo. Comité Olímpico Español. Centro Olímpico de Estudios Superiores. Madrid.

Olds, T., Tomkinson, G., Leger, L., \& Cazorla, G. (2006). Worldwide variation in the performance of children and adolescents: an analysis of 109 studies of the 20-m shuttle run test in 37 countries. Journal of Sports Science, 24(10), 1025-38.

Olmedo, J.A. (1998). Estrategias para aumentar el tiempo de práctica motriz en las clases de Educación Física escolar. Apunts Educación Física y Deportes 59, 22-30.

Organización Mundial de la Salud (2000). Obesity: Preventing and Managing. The Global Epidemic. Ginebra: Ediciones de la OMS.

Organización Mundial de la Salud, (2010). Recomendaciones mundiales sobre la actividad fisica para la salud. Ginebra: Ediciones de la OMS.

Pate, R.R., Davis, M.G., Robinson, T.N., Stone, E.J., Mckenzie, T.L., \& Young, J.C. (2006). Promoting physical activity in children and youth: a leadership role for schools: a scientific statement fromthe American Heart Association Council on Nutrition, Physical Activity, and Metabolism (Physical Activity Committee) in collaboration with the Councils on Cardiovascular Disease in the Young and Cardiovascular Nursing. Circulation, 114, 1214-1224.

Pieron, M. (2005). Para una enseñanza eficaz de las actividades fisico-deportivas (Vol. 132). Inde.

Revelles, A. B. F. (2008). El tiempo en la clase de educación física: la competencia docente tiempo. Deporte y actividad fisica para todos, (4), 102-120.

Ruiz, J.R., Castro-Piñero, J., Artero, E.G., Ortega, F.B., Sjöström, M., Suni, J., \& Castillo, M.J. (2009). Predictive validity of health-related fitness in youth: a systematic review. British Journal of Sports Medicine, 43(12), 909-923.

Sallis, J.F., Conway, T.L., Prochaska, J.J., Mckenzie, T.L., Marshall, S.J., \& Brown, M. (2001). The association of school environments with youth physical activity. American Journal of Public Health, 91, 618-620.

Sánchez-Cruz, J.J., Jiménez-Moleón, J.J., Fernández-Quesada, F. \& Sánchez, M.J. (2013). Prevalencia de obesidad infantil y juvenil en España en 2012. Revista Española de Cardiología, 66 (5), 371-376. doi: 10.1016/j.recesp.2012.10.016.

Solmon, M. (2003). Student issues in physical education classes: Attitudes, cognition, and motivation. En S. J. Silverman y C. D. Ennis (Eds.), Student learning in physical education: Applying research to enhance the instruction (pp. 147-164). Champaign.

Yanci Irigoyen, J., Vinuesa Mendieta, A., \& Yanci Irigoyen, L. (2016). El tiempo de compromiso motor en las sesiones de Educación Física del primer y segundo ciclo de Educación Primaria. Sportis, 2(2), 239-253.

Zabalza, M. A. (2016). El prácticum y las prácticas externas en la formación universitaria. Revista Prácticum, 1(1), 1-23. Recuperado de

http://revistapracticum.com/index.php/iop/article/download/15/42 